\author{
Abdellatif Chekir \\ Esprit School of Business \\ Lot $\mathrm{N}^{\circ} 05$, Zone industrielle Chotrana 11 , \\ 2083 Pôle Technologique El Ghazala \\ Ariana 2088, Tunisia \\ chqirlotfi@yahoo.fr \\ https://orcid.org/0000-0001-8135-5839
}

\title{
Calque linguistique et transfert sémantique
}

\begin{abstract}
Many idiomatic expressions specific to the French language have been translated into Arabic in favour of the contact between these two languages. However, these expressions cannot be easily located in Arabic since they receive a new texture, while taking advantage of this idiom's linguistic material. However, in order to stay faithful to the target language, these expressions should respect a few particular settings that are not fully respected, which makes recognizing them even harder. The semantic aspect added to the contextual reference allows us to identify this kind of calque.

Keywords: fixity, congruence, context, Idiomatic expressions, transfer
\end{abstract}

1. Introduction

Le contact étroit entre la langue française et la langue arabe en usage en Tunisie pendant la période coloniale et postcoloniale a eu un impact important sur le devenir de cet idiome à tous les niveaux Dans ce cadre, l'influence la plus notoire se manifeste plus particulièrement à travers l'emprunt et le calque. Cependant, ces deux phénomènes linguistiques ne présentent pas de fonctionnements similaires, 
n'adoptent pas les mêmes mécanismes de transfert et, par conséquent, n'offrent pas les mêmes procédés de reconnaissance puisque, contrairement à l'emprunt, le calque se manifeste en exploitant les matériaux linguistiques de la langue d'arrivée. C'est l'exemple de :

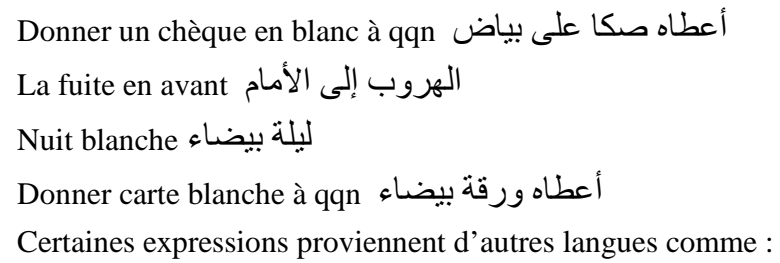

La langue de bois اللغة الخشبية, d'origine polonaise : drętwa mowa ;

Donner le feu vert, أعطاه الضوء الأخض , d'origine anglaise : To give the green light ${ }^{1}$.

Ce sont des expressions supranationales et qui se sont introduites en arabe par l'intermédiaire du français.

Cette texture particulière complique la tâche du chercheur qui tente de débusquer ce genre d'expression en l'absence de critères d'identification efficients. L'aspect sémantique et l'apport d'éléments contextuels pourraient assumer ce rôle et remédier à cette situation.

Notre travail cherche à démontrer que la composante sémantique associée à l'environnement contextuel peut jouer le rôle de pierre de touche qui permet de repérer le calque dans la langue arabe.

Pour ce faire, nous allons procéder à une distinction entre l'emprunt et le calque, distinction qui nous permet de montrer qu'ils ne disposent pas du même fonctionnement et par conséquent, ils n'exigent pas les mêmes critères de reconnaissance, critères que nous exposerons ensuite pour souligner leurs limites. Nous nous intéresserons par la suite aux spécificités du calque dans la langue arabe et plus particulièrement aux écarts que les expressions calquées opèrent par rapport aux patrons de départ. Sur cette base, nous essayerons de nous focaliser sur le volet sémantique, associé aux

\footnotetext{
${ }^{1}$ Les exemples sont puisés dans Chekir (2017).
} 
éléments contextuels, qui pourrait constituer un critère permettant de dépister les calques en arabe moderne.

\section{Les spécificités du calque}

\subsection{La distinction entre l'emprunt et le calque}

Le contact entre le français et l'arabe a eu une grande influence sur la langue arabe pratiquée en Tunisie et plus généralement, au Maghreb, qui est appelée à être enrichie et actualisée pour exprimer la modernité dans tous ses états. Cette influence est particulièrement perceptible au niveau du lexique, car la langue arabe a accueilli de nouveaux lexèmes et de nouvelles expressions empruntées à la langue française. Cependant, il est nécessaire de distinguer ces deux formes d'emprunt : l'emprunt au sens strict et le calque. L'emprunt consiste à intégrer une unité lexicale transférée à partir d'une autre langue telle quelle, avec son signifiant et son signifié moyennant une certaine adaptation au système morphologique et phonétique de la langue d'arrivée quand cela s'impose. Ainsi, le lexème emprunté peut être facilement détecté et reconnu comme un intrus puisqu'il affiche son origine étrangère d'autant plus qu'il ne résulte pas des mécanismes de la dérivation propres à la langue arabe. C'est l'exemple de yacht يخت, recyclage رسكلة Le calque, par contre, est un procédé qui exige la traduction littérale d'une expression figée à partir d'une autre langue avec plus ou moins de fidélité au patron d'origine. Il «consiste à former des mots ou des expressions en combinant des formes indigènes sur un modèle étranger »(Guiraud, 1968, p. 34). Autrement dit, le procédé du calque sollicite les matériaux linguistiques de la langue d'arrivée pour transférer les expressions préfabriquées. L'expression suit, par conséquent, tout un processus et passe par le filtre de la langue arabe pour s'y incruster. Se présentant ainsi à travers une nouvelle texture, l'expression occulte tout indice de son origine étrangère, brouille les pistes, devient difficile à détecter et échappe de ce fait à l'attention du chercheur. 
2.2. Le fonctionnement du calque

Le calque se présente ainsi comme un phénomène plus subtil que l'emprunt au sens strict, puisqu'il sollicite des compétences intellectuelles et des mécanismes cognitifs qui favorisent le transcodage, le passage d'un système linguistique à l'autre. Mais ce passage est semé d'embuches parce qu'il n'est pas question d'une simple traduction. L'opération est beaucoup plus complexe. Elle consiste à transférer une expression polylexicale, dont les unités entretiennent une relation particulière et constituent un ensemble solidaire. Elles ne peuvent par conséquent être appréhendées individuellement. L'expression calquée dans la langue cible doit également reproduire les contraintes formelles ainsi que le sens opaque du modèle de référence. Elle doit de plus être heureuse pour être adoptée par la communauté linguistique ; ce qui lui permet être intégrée dans le stock des expressions figées de L2. Mais quelles sont les étapes émigration.

\subsection{Les étapes de la genèse du calque}

Pour qu'une expression calquée puisse s'intégrer dans la langue d'arrivée et soit fixée dans l'usage, il faut qu'elle suive tout un processus de lexicalisation qui s'étale sur plusieurs étapes. Elle doit d'abord être transférée par un sujet bilingue et faire partie de son idiolecte, à la suite de son adaptation à son propre système linguistique. Dans une deuxième étape, ce locuteur doit l'intégrer dans l'échange verbal pour la rendre accessible à d'autres personnes et la faire accepter dans le discours avec son sens global. Elle doit, par conséquent, atteindre une fréquence massive à même de lui permettre de se greffer dans le système de la langue emprunteuse. En tout état de cause, un groupe relais assure au nouveau venu la propagation nécessaire qui lui permet d'avoir droit de cité et de jouir d'une certaine pérennité : école scientifique, mouvement littéraire et artistique, institution universitaire ou administrative, médias.

Ainsi l'expression calquée est codifiée comme n'importe qu'elle unité appartenant au lexique commun. Comme toute forme d'emprunt, elle subit tout un «mécanisme sémiotique par lequel le discours 
digère progressivement un corps étranger jusqu'à le codifier dans la langue »(Rey-Debove, 1998, pp. 152-153). Mais l'expression peut demeurer au stade de l'emploi individuel parce qu'elle n'a pas eu un tel écho auprès des usagers de la langue pour qu'elle devienne courante. Ainsi est-elle considérée comme un hapax et elle est vouée à l'oubli et à la disparition.

3. Les critères d'identification du calque

Cette identification semble très difficile, voire parfois impossible, parce que le calque est l'influence la moins perceptible qu'une langue puisse exercer sur une autre. Il se glisse subrepticement et se manifeste avec les ressources linguistiques propres à la langue réceptrice. Tout est fait pour camoufler l'origine étrangère de l'expression qui passe par le filtre de la langue arabe, s'infiltre, s'installe et s'incorpore dans le système linguistique porteur. Elle est même susceptible de subir une extension de sens ou d'être employée dans des contextes différents de ceux de l'expression de départ, comme n'importe quel lexème ou expression faisant partie du lexique arabe, à tel point qu'elle se fond dans la langue et devient méconnaissable pour un locuteur non averti, qui ne peut en aucune manière soupçonner son origine étrangère. Cette opération de détection des phrasèmes plus épineuse, lorsque leur assimilation dans la langue d'arrivée leur donne des apparences trompeuses, qui travestissent le moule d'origine par une modification quasi-complète de leurs configurations.

\subsection{Les caractéristiques du calque}

Quels sont les caractéristiques du calque ?

Ces expressions transférées en arabe doivent être investies de certaines caractéristiques analysées dans mon ouvrage (Chekir, 2019, pp. 117-118) pour être considérées comme des calques par la communauté linguistique : 
- elles doivent restituer la structure polylexicale propre au modèle d'origine ;

- les lexèmes qui constituent le calque doivent appartenir nécessairement à la langue d'arrivée ;

- le calque doit s'adapter généralement aux contraintes morphosyntaxiques propres à la langue d'arrivée ;

- le calque doit reproduire aussi bien la signification de l'expression de départ que les contraintes de cooccurrence des éléments surtout pour les collocations ;

- l'expression calquée doit résister à tous les types d'opérations syntaxiques : transformations, suppression, commutation ;

- l'expression calquée doit être massivement répandue dans l'usage pour être consacrée comme expression figée ou collocation à part entière dans la langue d'arrivée.

En d'autres termes, pour que l'expression soit codifiée comme calque, elle doit se comporter comme une expression figée à part entière en arabe afin d'enrichir le fonds commun des phrasèmes disponibles dans cette langue. Cependant, il est à remarquer que ces critères ne sont pas opératoires dans tous les cas de figures. Car certaines expressions restituent le patron de départ, alors que d'autres s'en écartent et deviennent méconnaissables.

\subsection{Calque fidèle et calque infidèle}

L'observation des calques suivants montre que la plupart des expressions sont fidèles, elles constituent une traduction littérale de leurs modèles d'origine et reproduisent intégralement leurs caractéristiques sémantiques et combinatoires. C'est l'exemple de

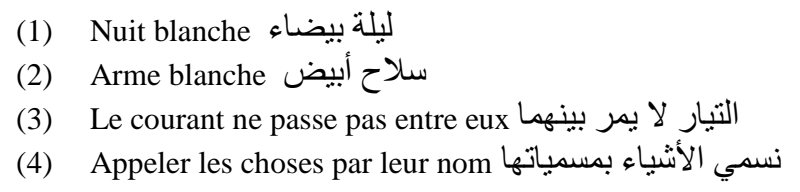

Ces expressions sont réfractaires à toutes les opérations de transformation, de substitution et d'insertion. 
D'autres expressions considérées comme des calques prennent une distance par rapport au modèle d'origine, en s'adaptant aux spécificités de la langue arabe ou en s'écartant du patron de départ. Mais cela défigure l'expression et accentue davantage la difficulté de la dépister. Nous pouvons observer ce phénomène d'écart à travers les exemples suivants :

(5) Mettre les points sur les وضع النقاط على الحروف Littéralement : Mettre les points sur les consonnes

(6) La bonne gouvernance الحوكمة الرشيدة

(7) La goutte qui a fait déborder le vase القطرة التي أفاضت الكأسة التسمة

Dans l'exemple (5), l'expression est adaptée, parce que la langue arabe ne transcrit pas les voyelles, même si elles sont prononcées à l'oral alors qu'elle distingue les consonnes par le nombre et la place des points autour de la consonne. Dans l'exemple (6) l'adaptation se fait par la postposition de l'adjectif après le verbe comme l'exige la norme de la langue arabe. Dans l'exemple (7) le verbe causatif polylexical, fait déborder, devient monolexical en arabe conformément aux mécanismes de la dérivation spécifiques à cette langue.

D'autres expressions calquées falsifient le patron de départ sans que cela ne soit exigé par les contraintes de la langue arabe. Nous pouvons le remarquer en observant les exemples suivants :

ذر الرماد على العيون) Jeter de la poudre aux yeux (8)

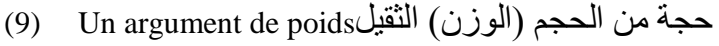

(10) Noyer le poisson تعويم القضية

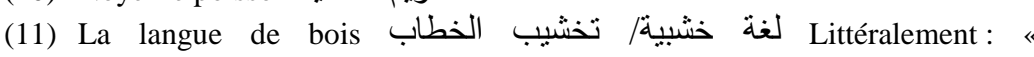
Boisification» du discours

Dans l'exemple (8) nous constatons que le calque ne reproduit pas littéralement l'expression car poudre a été traduit par رماد qui signifie حجم équivalent de «dimension» alors que l'exemple (10) recourt à la substitution de poisson par قضية signifiant «affaire », lexème puisé dans la définition de l'expression « obscurcir volontairement une 
affaire, de manière à embrouiller l'interlocuteur ». Dans l'exemple (11) l'adjectif خشبية devient un déverbal تخشيب.

L'écart est également perceptible au niveau du changement de contexte. Les expressions françaises sont généralement encodées dans des contextes linguistiques et extralinguistiques particuliers à même de permettre de les appréhender. Or, lorsqu'elles sont calquées en arabe, elles peuvent être actualisées dans des contextes différents. Nous illustrons cet écart par l'expression un argument de poids qui a pour équivalent en arabe حجة من الحجم (الوزن) الثقيل. Cette expression calquée peut être utilisée dans un contexte différent en arabe comme dans l'exemple suivant :

qui signifie littéralement « une intervention d'une dimension lourde ».

Nous pouvons postuler que ce qui est figé en arabe, c'est plutôt من «d'une dimension lourde». En d'autres termes, nous sommes en présence, dans ce cas de figure, d'un exemple de réanalyse, puisque les limites de la séquence figée ont été perçues différemment en arabe.

Ces écarts s'expliquent par la nature du transfert, car les locuteurs bilingues procèdent à une traduction spontanée, qui ne se réfère à aucune méthodologie mûrement réfléchie, fixée au préalable comme pour la traduction terminologique.

Cependant, malgré ces différents types d'entorses structurelles et combinatoires et contextuelles, ces expressions calquées reproduisent le sens global du modèle d'origine.

Mais comment détecter ces calques en l'absence de critères efficients et de méthodes sophistiquées surtout que le transfert se fait entre deux langues qui n'appartiennent pas à la même famille et qui ne grammatisent pas les phénomènes de la même manière ?

L'aspect sémantique associé au contexte immédiat peut constituer un critère opérant dans ce genre de tentatives. 
4. Le critère sémantique et le contexte d'emploi

Les critères morphologique, syntaxique et étymologique ne peuvent être opératoires pour l'identification des calques dans le cadre du transfert des expressions du français vers l'arabe parce que beaucoup de calques ne reproduisent pas fidèlement la structure de l'expression française ni le même contexte d'emploi, d'autant plus que ces deux langues n'adoptent pas le même alphabet ni les mêmes mécanismes morphologiques. Nous pouvons recourir au critère sémantique vu que le signifié de l'expression est restitué au cours du passage vers L2.

Ce critère nous semble le plus pertinent pour découvrir les expressions figées calquées étant donné qu'elles manifestent en général un certain degré d'opacité au niveau sémantique même si cet aspect, n'ayant pas de consistance matérielle, n'est pas tangible. Autrement dit, généralement le sens de ces séquences n'est pas compositionnel, ce qui peut dérouter le locuteur non averti. Nous pouvons le vérifier à travers des phrases comme :

(12) وصلنا إلى عنق الزجاجة Littéralement : nous sommes arrivés au goulot d'étranglement

تمثل هذه الفكرة حجر الزاوية لنظرية ابن رشد Littéralement : cette idée représente la pierre angulaire de la théorie d'Averroès

(14) أعطاه ورقة بيضناء Littéralement : Il lui a donné une carte blanche.

est l'équivalent de goulot d'étranglement, dans la phrase (12) et équivaut à pierre angulaire dans la phrase (13), alors que la phrase 14 constitue une traduction littérale de toue la phrase française.

Ces expressions n'appartiennent pas au stock de phrasèmes spécifiques à la langue arabe. Par conséquent, ces phrases sont illisibles après une lecture au premier degré par un locuteur arabophone parce que leur sens n'est pas compositionnel et ne peut ainsi être saisi après un calcul du sens. Ce manquement par rapport aux règles de la congruence et de la combinaison des lexèmes sur l'axe syntagmatique bloque l'interprétabilité de la phrase et peut interpeller le destinataire du message parce que les mécanismes 
habituels du décodage ne lui permettent pas d'associer les unités lexicales pour appréhender le sens de la phrase même en sollicitant le contexte linguistique et extralinguistique. Autrement dit, le parcours interprétatif ne peut être d'aucun secours pour appréhender l'expression. Ainsi cette opacité de l'expression doublée de l'incongruence sémantique peut constituer un paramètre de reconnaissance des calques, parce que ce type de phrases, qui semblent inacceptables au premier abord, suscitent la curiosité des chercheurs. Ceux-ci essayeront de comprendre la signification de l'expression, en observant les contraintes de son emploi et de retrouver son origine, ainsi que les glissements qu'elle a subis. Mais cet effort ne peut être mené à bien qu'après une analyse en profondeur du mécanisme de ces expressions lequel permet de passer au crible leur structure syntaxique et leur charge sémantique, tout en procédant à un retour aux dictionnaires de phraséologie consacrés.

\section{Conclusion}

Le calque peut constituer un moyen pour enrichir le stock d'expressions préfabriquées disponibles en arabe. Une fois intégrées au système, ces expressions calquées peuvent être encodées comme n'importe quelle expression spécifique à la langue arabe. Mais en passant par le filtre de la langue arabe ces expressions perdent leur fixité qui constitue une de leurs caractéristiques définitoires. Elles sont déconstruites. Elles suivent un nouveau processus d'ajustement et de figement qui aboutira à leur fixation. Avec cet habillage nouveau, elles occultent leur origine et deviennent méconnaissables et indétectables en l'absence de méthodes de repérage sophistiquées. Cependant, l'aspect sémantique, associé aux éléments contextuels, peut être d'un grand apport pour dépister ces calques toutefois c'est une opération qui demeure très complexe au regard des écueils sur lesquels nous butons.

\section{Bibliographie}

Chekir, A. (2017). Dictionnaire des calques français arabe. Collocations et expressions figées. Tunis: Centre de Publication Universitaire. 
Chekir, A. (2019). Le calque linguistique en arabe moderne. Tunis: Centre de Publication Universitaire.

Guiraud, P. (1968). Les mots étrangers. Paris: Presses Universitaires de France.

Rey-Debove, J. (1998). La linguistique du signe. Paris: Armand Colin. 